\title{
The First Fermi-LAT SNR Catalog and Cosmic Ray Implications
}

\author{
F. de Palma ${ }^{1,2, a}$, T. J. Brandt ${ }^{3}$, and J.W. Hewitt ${ }^{4}$ on behalf of Fermi-LAT Collaboration \\ ${ }^{1}$ Istituto Nazionale di Fisica Nucleare, Sezione di Bari \\ ${ }^{2}$ Universitá Telematica Pegaso, Piazza Trieste e Trento, 48, 80132 Napoli, Italy \\ ${ }^{3}$ NASA/Goddard Space Flight Center \\ ${ }^{4}$ University of North Florida
}

\begin{abstract}
While supernova remnants (SNRs) are widely thought to be powerful cosmicray accelerators, indirect evidence comes from a small number of well-studied cases. Here we systematically determine the gamma-ray emission detected by the Fermi Large Area Telescope (LAT) from all known Galactic SNRs, disentangling them from the sea of cosmic-ray generated photons in the Galactic plane. Using LAT data we have characterized the 1-100 GeV emission in 279 regions containing SNRs, accounting for systematic uncertainties caused by source misattribution and instrumental response. We classified 30 sources as SNRs, using spatial overlap with the radio emission position. For all the remaining regions we evaluated upper limits on SNRs' emission. In the First Fermi-LAT SNR Catalog there is a study of the common characteristics of these SNRs, such as comparisons between $\mathrm{GeV}$, radio and $\mathrm{TeV}$ quantities. We show that previously satisfactory models of SNRs' GeV emission no longer adequately describe the data. To address the question of cosmic ray (CR) origins, we also examine the SNRs' maximal CR contribution assuming the $\mathrm{GeV}$ emission arises solely from proton interactions. Improved breadth and quality of multiwavelength (MW) data, including distances and local densities, and more, higher resolution gamma-ray data with correspondingly improved Galactic diffuse models will strengthen this constraint.
\end{abstract}

\section{The SNR catalog}

Using three years of LAT data starting from 279 SNRs' radio spatial information [1] we detected 102 candidates with a significance above $5 \sigma$. 36 of them passed our spatial association probability threshold. For those sources we evaluated their spectral and spatial characteristics with systematic and statistical uncertainties. The latter are obtained summing in quadrature the uncertainty in the effective area and the effects of changing the interstellar emission model (IEM), which accounts for $\gamma$-rays produced by CR interactions with interstellar gas and radiation fields in the Milky Way. We used eight alternative IEMs from [5] for more details see appendix B of [2] and [3].

Of these 36, 30 SNRs show significant emission for all alternative IEMs and are classified as likely GeV SNRs. Of the remaining six, four were identified as sources which are not SNRs; while the other two candidates were considered marginal candidates due to their dependence on the IEM. Of the 30 sources likely to be GeV SNRs, 17 show evidence for extension $\left(\mathrm{TS}_{\text {ext }}>16\right)$. Only sources

\footnotetext{
a e-mail: francesco.depalma@ba.infn.it
} 
associated with SNRs G34.7-0.4 and G189.1+3.0 are fit with $\log P$ spectra instead of the usual powerlaw (PL) spectra. Of the classified candidates, four extended and 10 point SNRs are new. Other 14

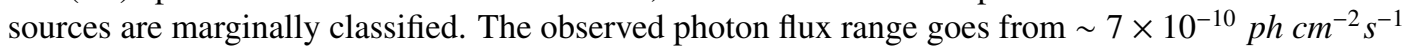
to $\sim 7 \times 10^{-8} \mathrm{ph} \mathrm{cm}^{-2} \mathrm{~s}^{-1}$, while the PL index range goes from 1.5 to $\sim 4.0$.

We, also, evaluated the chance of spatial coincidence probability using a mock catalog for both the candidates and the marginally classified candidates. We reported also 245 bayesian [4] upperlimits (UL) for all the non detected radio SNRs.

\section{Multiwavelength correlations}

We have studied the relationship between the newly measured $\mathrm{GeV}$ indices and radio and $\mathrm{TeV}$ spectral indices from literature, as shown in Fig. 1. Nearly all candidates have $\gamma$-ray photon indices that are softer than predicted given their radio spectra, regardless of the possible $\mathrm{GeV}$ emission mechanisms. The three young SNRs in blue are consistent with a single underlying particle population, and it has been suggested inverse Compton emission (dashed line in the figure) at $\mathrm{GeV}$ energies. The observed soft $\mathrm{GeV}$ spectra relative to the radio has several potential explanations. The underlying leptonic and hadronic populations may have different PL indices. The emitting particle populations may may follow a broken PL or even differing spectral shapes. Or, there may be different zones with different properties dominating the emission at different wavelengths.

Six of the ten SNR candidates have TeV indices softer than their GeV indices, while three have $\mathrm{GeV}$ and $\mathrm{TeV}$ indices consistent with each other, within statistical and systematic errors, as shown in Fig. 1. Such a change of the spectral index from $\mathrm{GeV}$ to $\mathrm{TeV}$ suggests that another particle population may dominate at higher energies or that the emission mechanism may change between the $\mathrm{GeV}$ and $\mathrm{TeV}$ regimes.

\section{Constraining Cosmic Ray acceleration}

SNRs have long been held the most promising candidate sources of Galactic CRs, capable of supplying the flux observed at Earth if their efficiency in accelerating CR protons and nuclei stands between $\sim 5-10 \%$. In this section we would like to understand if our detected SNR population is compatible with the observed CRs flux and spectra. In the following, we assume that the $\gamma$-ray emission from SNRs probed with LAT entirely arises from the interaction of CR protons and nuclei with the surrounding ISM or circumstellar medium through the production and subsequent decay of $\pi^{0}$. Given that two other emission mechanisms involving accelerated leptons, namely non-thermal bremsstrahlung and inverse Compton scattering, could also contribute in the $\gamma$-ray domain, the constraints derived from the LAT measurements should be considered as upper limits on the CR energy content in SNRs. As seen in [2] and references therein the expected $\gamma$-ray flux is nearly independent of the CR maximal energy as long as $E_{\mathrm{CR} \text {, max }} \gtrsim 200 \mathrm{GeV}$ and $\Gamma_{\mathrm{CR}} \gtrsim 2$. In this case, it can conveniently be approximated using the following expression:

$$
F(1-100 \mathrm{GeV}) \approx f\left(\Gamma_{\mathrm{CR}}\right) \times \frac{\epsilon_{\mathrm{CR}}}{0.01} \times \frac{E_{\mathrm{SN}}}{10^{51} \mathrm{erg}} \times \frac{n}{1 \mathrm{~cm}^{-3}} \times\left(\frac{d}{1 \mathrm{kpc}}\right)^{-2} \times 10^{-9} \mathrm{~cm}^{-2} \mathrm{~s}^{-1}
$$

where $f\left(\Gamma_{\mathrm{CR}}\right)$ is such that $f(2.0)=2.06, f(2.5)=1.07$, and $f(3.0)=0.34$. Using in the previous equation the $\gamma$-ray flux, density and distance of each SNR we can determine its maximal CR energy content $E_{\mathrm{CR}}$ as shown in Fig. 2. Distances and effective densities are taken from the literature when available, see [2]. We use the canonical value of $10^{51} \mathrm{erg}$ for $E_{\mathrm{SN}}$. 

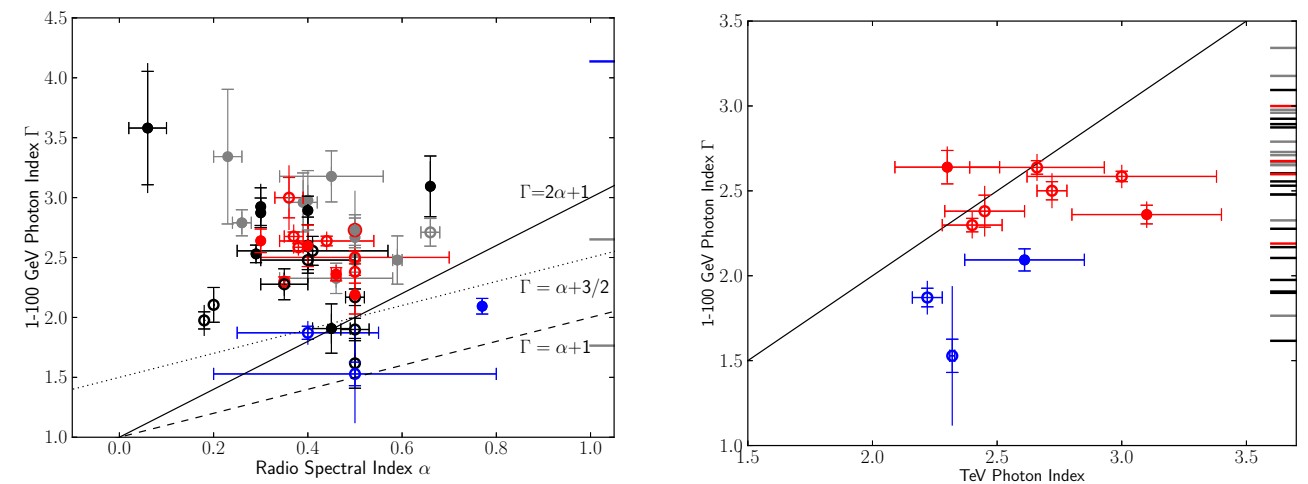

Figure 1: Open circles indicate extended SNRs while filled circles indicate point-like sources. All SNRs that passed classification criteria are shown as black unless also classified as young non-thermal $\mathrm{X}$-ray SNRs (blue) or as interacting with molecular clouds (red). Marginally classified candidates which did not pass classification but still had both fractional overlaps $>0.1$ are grey. If they are also young or interacting, they are outlined in blue or red, respectively. Statistical error bars have caps; error bars without caps present the systematic error. Ticks along the right hand side of the right plot show the $1-100 \mathrm{GeV}$ photon indices of those SNRs without reported radio or TeV spectral indices. Left: Comparison of radio spectral index, $\alpha$, and $\mathrm{GeV}$ photon index, $\Gamma$. The expected correlations for $\pi^{0}$ decay or $\mathrm{e}^{ \pm}$bremsstrahlung (solid) and inverse Compton (dashed) are plotted. Right: GeV index compared to published $\mathrm{TeV}$ spectral indices. The line corresponds to equal index values.

As is clearly visible for the first two subclasses of SNRs, the estimates and upper limits on the CR energy content span more than three orders of magnitude, from a few $\times 10^{49}$ erg to several $\times 10^{52} \mathrm{erg}$. For the interacting SNRs that lie above the $\epsilon_{\mathrm{CR}}=E_{\mathrm{CR}} / E_{\mathrm{SN}}=1$ dashed line, the densities experienced by the CR particles in the molecular cloud interaction region are likely much larger than those used in this analysis. Interacting candidates' lying above this limit suggests that they are likely the sites of hadronic interactions in dense environments. In contrast, most of the young SNRs lie at or below this luminosity limit, suggesting that IC processes may contribute to their measured luminosity. New information about distances and densities can give us further insight on the possibility of known SNRs to provide the observed galactic CRs. The usual assumption of $\epsilon_{\mathrm{CR}}=0.1$, required for the Galactic SNR population to supply the CR flux observed at Earth, is compatible with the results of the FermiLAT SNR catalog, for more details see [2].

\section{Conclusion}

In this work we have studied all known radio SNRs in the $\gamma$-ray band and the correlation of our results with spectral and spatial information from radio and $\mathrm{TeV}$ bands. This was done in order to understand if the underlying CR population, possibly responsible of these emission, is the same and has the same spectral characteristics. Within the limits of existing MW data, our observations generally support previous findings of changes in spectral slope at or near $\mathrm{TeV}$ energies and a softening and brightening in the $\mathrm{GeV}$ range with age, yet we see indications that new candidates and new MW data may provide evidence of exceptions to this trend. 


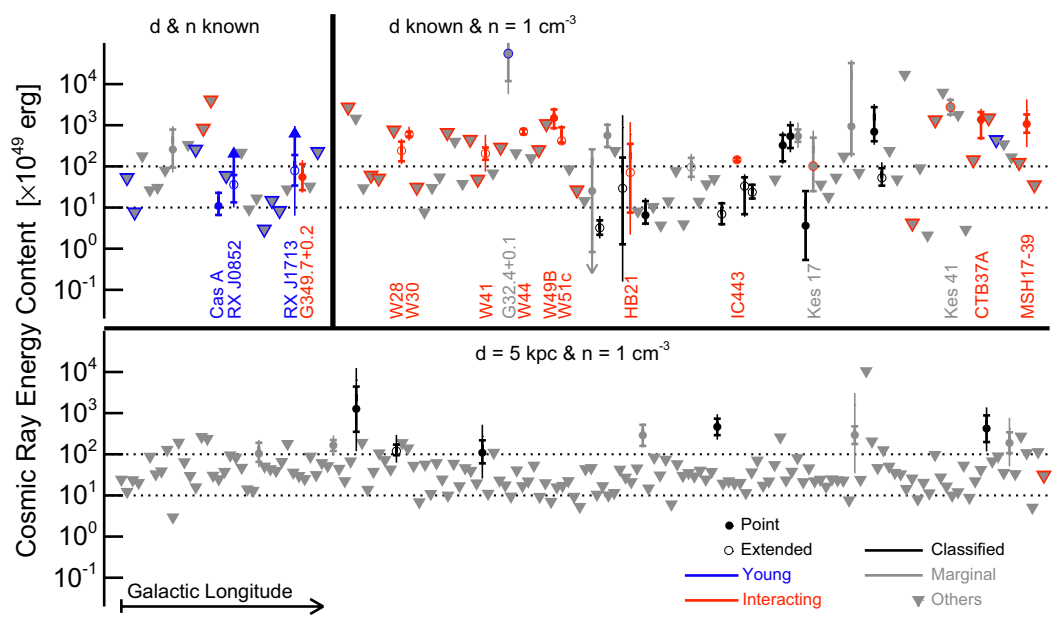

Figure 2: Estimates of the CR energy content for all Galactic SNRs, divided into three categories each sorted by Galactic longitude: SNRs with existing distance and density estimates (upper left panel); SNRs with known distances (upper right panel); and SNRs with unknown distance and density (lower panel).The two dashed lines indicate a CR energy content of 10 and $100 \%$ of the standard supernova (SN) explosion energy.

We find, also, that the limits on CR energy content span more than three decades, including many interacting candidates for which the densities in the interaction regions are much greater than the nominal density assumed in the calculation, and young candidates with efficiencies below the nominal $\sim 10 \%$, consistent with possible leptonic emission predictions (e.g. IC). The contribution from all SNRs, particularly those with flux upper limits, constrains the energy content put into CRs from the known SNRs to less than 10\%, particularly in regions of well characterized IEM background. We find that the candidates and upper limits are generally within expectations if SNRs provide the majority of Galactic CRs.

\section{Acknowledgments}

The Fermi-LAT Collaboration acknowledges support for LAT development, operation and data analysis from NASA and DOE (United States), CEA/Irfu and IN2P3/CNRS (France), ASI and INFN (Italy), MEXT, KEK, and JAXA (Japan), and the K.A. Wallenberg Foundation, the Swedish Research Council and the National Space Board (Sweden). Science analysis support in the operations phase from INAF (Italy) and CNES (France) is also gratefully acknowledged.

\section{References}

[1] Green, D. A. 2009, Bulletin of the Astronomical Society of India, 37, 45

[2] Acero, F. et al. 2016, ApJS, 224, 8

[3] de Palma, F. et al. arXiv:1304.1395

[4] Helene, O. 1983, Nuclear Instruments and Methods in Physics Research, 212, 319

[5] Ackermann, M. et al. 2012, ApJ, 750, 3 